\title{
Agradecimento a pareceristas do v. 16, n. 2, dezembro 2020
}

Acknowledgment to the referees of Vol. 16, Issue 2, December 2020

Agradecemos a colaboração neste número dos pareceristas Adilson Vaz Cabral Filho, Alberto Calil Junior, Ana Ligia Medeiros, Ana Maria Barcellos Malin, André Appel, Anne Clinio, Arthur Bezerra, Beatriz Martins, Bethânia Almeida, Bruno Henrique Alves, Carla Baiense Felix, Carlos Alberto Ávila Araújo, Claudia Chamas, Claudio Marcondes Castro Filho, Diego Bevilaqua, Eliane Bezerra Paiva, Eloísa da Conceição Príncipe de Oliveira, Emir Suaiden, Fábio Gouveia, Fátima Martins, Fernanda Lopes Fonseca, Fernanda Lima, Gabriela da Silva Zago, Gabrielle Tanus, Gilda Olinto, Giuseppe Cocco, Gustavo Henrique de Araújo Freire, Henrique Parra, lara Vidal, Igor Sacramento, Isa Maria Freire, Ivan Paganotti, Izabel França de Lima, Janaina Oliveira Pamplona da Costa, Janicy Aparecida Pereira Rocha, Jorge Machado, Kizi Mendonça de Araújo, Lea Maria Leme Strini Velho, Leandro Nunes de Castro Silva, Lena Vania Ribeiro Pinheiro, Luana Rocha, Luca Schirru, Marcelo Fornazin, Márcia Feijão, Márcio Castilho, Marco Antonio de Almeida, Marco Antonio Roxo da Silva, Marco Schneider, Maria Cristiane Barbosa Galvão, Maria Nélida González de Gómez, Marianna Zattar, Marta Leandro da Mata, Meri Nádia Marques Gerlin, Michelli Costa, Natália Elvira Sperandio, Patricia Bertin, Patrícia Porto, Paulo César Castro, Paulo Fonseca, Priscila Gonzalez, Raul Murad, Regina Marteleto, Ricardo Jorge de Lucena Lucas, Ricardo Pimenta, Rodrigo Moreno Marques, Rodrigo Piquet Saboia de Mello, Ronaldo Ferreira de Araujo, Thaiane Moreira de Oliveira, Thiago Magela Rodrigues Dias, Vanessa Ribeiro Corrêa Sampaio Souza, Vanessa Schottz Rodrigues, Vitor de Azevedo Almeida Junior, Viviane Veiga e Welder Antonio Silva.

Os Editores 